\title{
Distance Between Two Snaked-lay of Subsea Pipeline
}

\author{
Yuxiao Liu $^{1, a^{*}}$ \\ 1,Dept. of Management Since and Engineering,Shandong Institute of Business and \\ Technology, Yantai, China \\ ae-mail:63563298@qq.com
}

\begin{abstract}
Keywords:snake lay; lateral buckling; subsea pipeline; high pressure/high temperature.
Abstract. Global buckling for exposed HPHT (High Pressure / High Temperature) subsea pipelines is an important feature that needs to be considered during detailed design. The design of exposed pipelines will aim for; either to document that the pipeline will not buckle laterally, or, that the pipeline will buckle laterally and the post-buckling condition is acceptable. By safely triggering controlled buckles at predetermined locations, snaked lay design is often the preferred method to manage lateral buckling. Using finite elemet analyses, This paper discusses the effect of distance between two snaked-lays on lateral deflection, bending moment and strains. It was found that the distances between two snaked-lays have small effect on the max bending moment of the snaked-lay pipeline. For both sine curve and arc curve, with distances between two snaked-lays increasing, the resulting axial plastic strain is clearly enhanced.
\end{abstract}

\section{Introduction}

Pipelines operating at temperatures and pressures above ambient will tend to expand, due to thermal and pressure loading. Expansion along the flow line length is restrained by axial friction between the pipe and seabed causing axial compressive forces to develop in the flow line system. This force is relieved towards the pipeline ends, if the pipeline is free to expand ${ }^{[1]}$. If the global buckling occurrs, it has to be established whether the resulting load within the buckle is acceptable if only one buckle forms. A widely used method to ensure that the maximum allowable buckle loads are not exceeded is to ensure that several buckles will form so that the available thermal expansion will be shared by several buckles rather than localized in a single buckle ${ }^{[1]}$. An additional consideration if multiple buckle sites are adopted as a design solution, is to ensure that buckles will be triggered as intended at each buckle site. There is a danger if the maximum distance between each buckle site is too short; It might cause interference between adjacent sites, i.e. one buckle will in effect suppress the buckle at the adjacent buckle site.

This paper describes the distance between two snaked-lay, several FE analyses are performed in this paper to show that the influences of distance between two snaked-lay, length of imperfection(L) and $\operatorname{radii}(\mathrm{R})$.

\section{Snaked-lay General Description}

Figure 1 presents a typical lateral snake lay configuration, and the curved section is sine curve as shown in Figure 2.

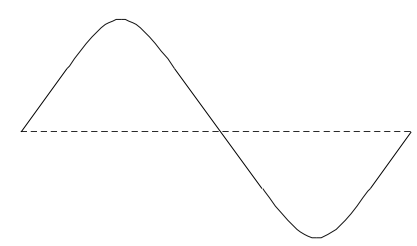

Fig. 1 Snaked Lay Configuration

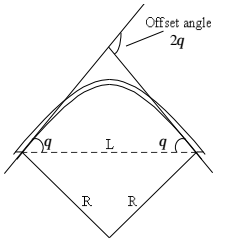

Fig. 2 Curved Section

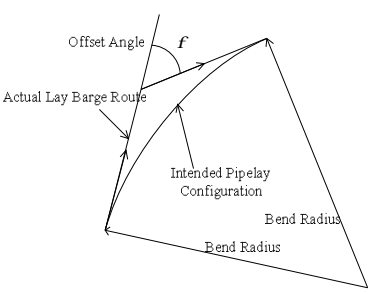

Fig. 3 Curved Section

In Figure 2, sine length、 lay radius and offset angle satisfy the following relationship. 


$$
\sin \theta=\frac{L}{2 R}
$$

Where $\theta$ is offset angle, $R$ is lay radius, $L_{0}$ is the sine length.

In this paper arc curve (as following) is studied too, the relationship between the arc length[2], pipelay offset angle and radius is shown in Fig. 3 and is expressed by equation 1 below.

$$
L_{\text {Arc }}=R \phi
$$

Where: $L_{A r c}$ is Arc Length; $R$ is Bend Radius; $\phi$ is Offset Angle.

\section{Finte Element Analysis of Snaked Lay Pipeline}

Pipeline and soil parameters are shown in Table 1.

Table1 Pipe and Soil Parameters

\begin{tabular}{|c|c|c|}
\hline Parameter & Value & Units \\
\hline Outside Diameter & 814 & $\mathrm{Mm}$ \\
\hline Steel Wall Thickness & 25.4 & $\mathrm{~mm}$ \\
\hline Submerged Weight & 3386 & $\mathrm{~N} / \mathrm{m}$ \\
\hline Axial Friction Factor & 0.5 & \\
\hline Lateral Friction Factor & 0.87 & \\
\hline
\end{tabular}

Pipeline Model

The pipeline was modeled using 2 noded, 6 degrees of freedom PIPE20 elements which have plastic capability. The material behavior was defined by the Ramberg, 2. and Osgood, W.R.(1943) stress-strain constitutional relationship. The derated values for the yield and tensile stresses at design temperature were used throughout the analysis.

Only symmetrical modes are analyzed and therefore only one half of the pipeline was modeled with symmetry boundary conditions specified at the start end of the model.

Soils Model

Non-linear ideal elasto-plastic soil axial and lateral friction behavior was modeled using the COMBIN39 spring element. The displacement at which full is mobilized was defined to be $2 \mathrm{~mm}$ simulating the behavior of a stiff soil. One spring in the axial and one in the lateral direction were specified at each pipe node. The seabed was modeled as a flat seabed ignoring spanning parts of the pipeline. The pipeline was pinned in the vertical direction at each pipe node. The force-displacement relationship is illustrated in Fig.4. The relationship between yield force, friction coefficient and submerged weight along pipeline per unit can be expressed as:

$$
F=\mu W
$$

Where, $F$ is yield force; $\mu$ is friction coefficient between seabed and pipeline; $W$ is submerged weight along pipeline per unit.

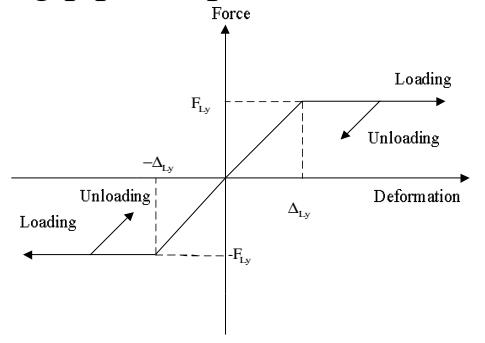

Fig. 4 Model for Soil

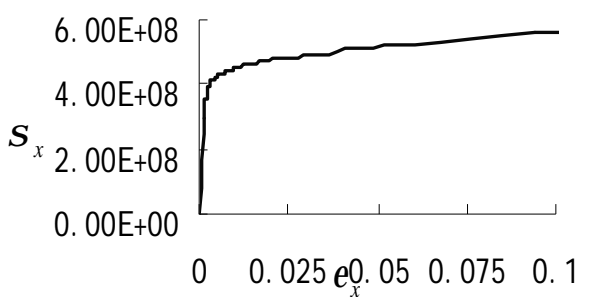

Fig. 5 Stress-Strain Relationship For X65 Pipeline Steel Changing distances between two snaked-lays ( $\mathrm{L}=200, \mathrm{R}=2500)$ was performed.

ANSYS is used to study lateral buckling of snaked-lay pipeline. The pipeline is modeled as PIPE20 elements with plastic capability. The stress-strain constitutional relationship is shown in Fig.5. The expression of the Ramberg-Osgood model is: 


$$
\varepsilon_{x}=\frac{\sigma_{x}}{E_{0}}\left[1+\frac{n}{1+r}\left(\frac{\sigma_{x}}{\sigma_{y}}\right)^{r}\right]
$$

where $\varepsilon_{x}$ and $\sigma_{x}$ are the engineering strain and stress; $E_{0}$ is the initial Young's modulus; $\sigma_{y}$ the yield stress of pipe material; $n$ and $r$ are the Ramberg-Osgood parameters.

Pipeline-soil interaction model

Non-linear ideal elasto-plastic soil axial and lateral friction behavior was modeled using the COMBIN39 spring element[2-6], as Fig.6 shows. As only lateral buckling is of interest in this paper, the seabed was modeled as a flat seabed ignoring spanning parts of the pipeline. The pipeline was pinned in the vertical direction at each pipe node. Finite Element Model of pipeline is shown in Fig.7

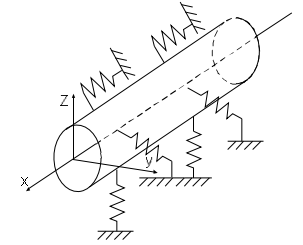

Fig. 6 Pipeline-soil interaction model

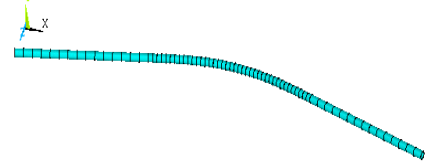

Fig. 7 Pipeline finite element model

Changing distances between two snaked-lays

Figure 8 shows moment distribution of snaked-lay pipeline with changing distances between two snaked lays. It shows clearly that the max bending moments of snaked-lay pipeline are enhanced with the distance between two snaked-lays increasing, but from the figure it can be seen that the increment is small.

The corresponding max bending moments of snaked-lay pipeline with arc cure show the same tendency.

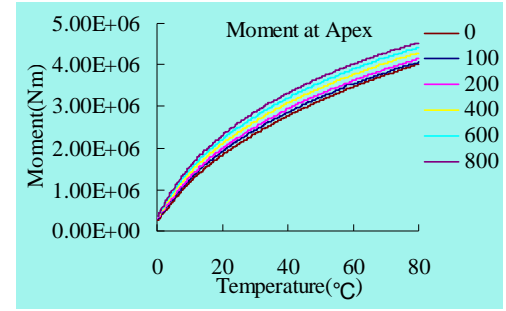

Fig. 8 Bending Moments vs. Temperature (sine curve)

Figure 9 shows the lateral deflection of the buckle apex relative to the initial curved configuration with the distance between two snaked-lays changing from $0 \mathrm{~m}$ to $800 \mathrm{~m}$, and Figure 10 is the left snaked-lay lateral deflections. The comparison between initial snaked-lay and deformed shape of pipeline is shown in Figure 11. From Figure 9 and Figure 10 it can be seen that the buckle forms at around the apex of the planned buckle site.

The result of arc curve is presented in Figure 12 and Figure 13. Compared with sine curve it can be seen that the center of the snake lay amplitude occur offset from the apex of the snake lay geometry. But with distance increasing the offset is reduced.

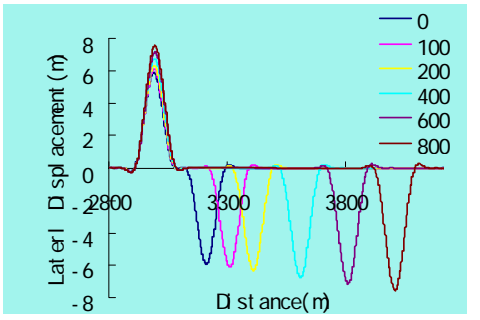

Fig. 9 Lateral Deflections along Pipeline

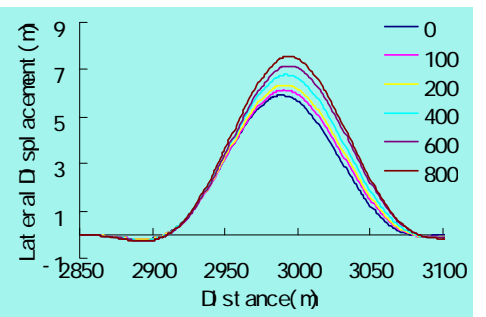

Fig. 10 Lateral Deflections along Pipeline 


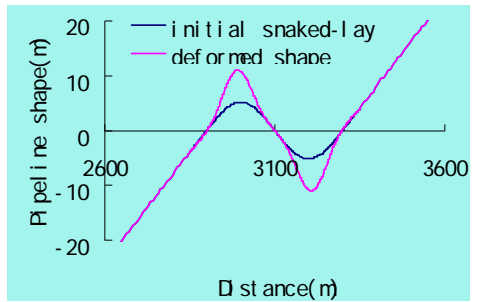

Fig.11 Pipeline Shape

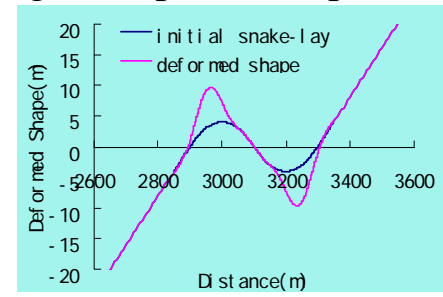

Fig. 13 Pipeline Shape

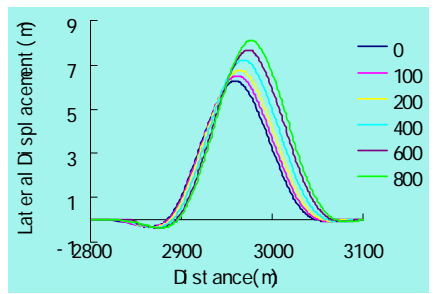

Fig.12 Lateral Deflections along Pipeline

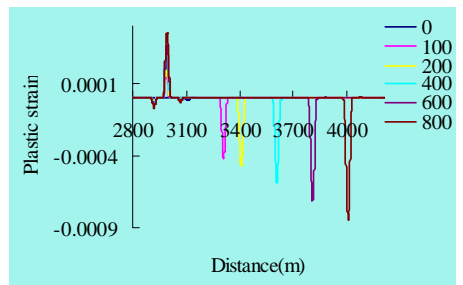

Fig. 14 Axial Plastic Strain Distributions

Axial plastic strain along the pipeline is presented in Figure 14. By gradually increasing distance between two snaked-lays to $800 \mathrm{~m}$, the resulting axial plastic strain is clearly enhanced. The corresponding axial plastic strain of arc curve shows the same tendency, but for the same distance the plastic strain of arc curve is larger than the plastic strain of sine curve.

\section{Conclusions}

This paper discusses the effect of distance between two snaked-lays on lateral deflection, bending moment and strains. The main findings are as follows:

For both sine curve and arc curve, the distances between two snaked-lays have small effect on the max bending moment of the snaked-lay pipeline; With distances between two snaked lays changing, the buckle of sine curve forms at around the apex of the planned buckle site, but for arc curve the center of the snake lay amplitude occur offset from the apex of the snake lay geometry, and with distance increasing the offset is reduced; For both sine curve and arc curve, with distances between two snaked-lays increasing, the resulting axial plastic strain is clearly enhanced.

\section{Acknowledgment}

This research is financially supported by the Humanity and Social Science Youth foundation of Ministry of Education (13YJCZH114).

\section{References}

[1] DNV-RP-F110. Global buckling of submarine pipelines structural design due to high temperature/high pressures. Det Norske Veritas, Norway, 2007.

[2] Rathbone, A.D. Rundsag, J.O., Tornes, K., Cumming, G., Roberts, C., effect of lateral pipelay imperfections on global buckling design, ISOPE 2008

[3] Jiong Guan, Per R. Nystr $\phi$ m, Hans F. Hansen, optimized solutions to control lateral buckling of pipelines with snaked-lay: theoretical and numerical studies, OMAE 2007

[4] J.O. Rundsag, J P Kenny Norge AS, Stavanger, Norway, optimized snaked lay geometry, ISOPE 2008

[5] J. Hooper, E. Maschner, T. Farrant, JP Kenny Ltd, HT/HP Pipe-in-Pipe Snaked Lay Technology-Industry Challenges, OTC 2004 
Lars Christensen, Perth, Displacement Control in Lateral Buckling of "Short" Pipelines. Proceedings of the FifteenthInternational Offshore and Polar Engineering Conference, 2005. 